\title{
Incorporating a generalised additive model of river nutrient concentrations into a mechanistic receiving water model
}

\author{
Barbara J. Robson and Vincent Dourdet \\ CSIRO Land and Water, Black Mountain \\ Email: barbara.robson@,csiro.au
}

\begin{abstract}
Reefs is a large, collaborative project that is building catchment and marine models for Australia's Great Barrier Reef Lagoon (GBRL), a world-heritage environmental asset. The eReefs package includes three-dimensional mechanistic biogeochemical, sediment and hydrodynamic models for the entire GBRL on $4 \mathrm{~km}$ and $1 \mathrm{~km}$ grid scales, along with a relocatable coastal and estuary model (RECOM) that can be nested within the larger-scale models. Source Catchment models developed by the Government of Queensland for each GBRL catchment will be used to run scenarios to predict the effects of management and land use changes on nitrogen, phosphorus and sediment loads reaching each river. For day-to-day near-realtime and forecast-mode running of the marine models, however, another approach is needed to provide the river loads of sediments, dissolved and particulate loads required as boundary conditions.
\end{abstract}

Generalised Additive Models (GAMs) have been shown (e.g. Kuhnert et al., 2012) to be powerful tools for the prediction of suspended sediment and particulate nutrient loads in tropical rivers. Here, we extend previous work to build GAMs that are able to predict concentrations of suspended sediments, dissolved and particulate nutrients in the Fitzroy River (Queensland) on a daily time-step.

In developing the GAMs, we tested a number of routinely and frequently measured meteorological and hydrological variables for potential predictive power. The new terms considered included water temperature (which may alter biogeochemical processing rates), air temperature (a more reliably measured proxy for water temperature), electrical conductivity (which may reflect the influence of particular subcatchment sources), barometric pressure (an indicator of local storm activity), wind stress (which may affect resuspension and mixing in the river and its weirs) and flow from river tributaries (a direct measure of the influence of particular subcatchments). The models generated were tested with regard to the validity of key statistical assumptions, and were then validated against a subset of observational data that had been held back from the original calibration.

The strongest models included flow in the Fitzroy River, flow in one or more tributaries, and a discounted flow term that reflected flow in the preceding days and weeks. Models that did not include tributary flow were able to predict concentrations of particulate, but not dissolved materials. Neither meteorological terms nor electrical conductivity proved to be useful predictors, while water temperature was of marginal value.

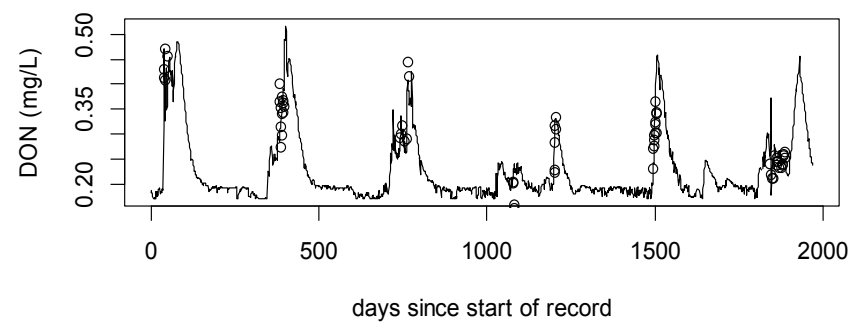

Figure 1 Time-series of dissolved organic nitrogen (DON) from the GAM for input to marine models. Observations are shown as dots.

The final GAM provide more accurate predictions on a daily time-step than previously available methods, for both dissolved and particulate materials, and is being used to provide time-series input (e.g. Figure 1) to mechanistic marine models.

Keywords: $\quad$ eReefs, dissolved nitrogen, dissolved phosphorus, statistical model, nutrient loads, sediment loads 
Robson and Dourdet, Incorporating a generalised additive model of river nutrient concentrations into a mechanistic receiving water model

\section{INTRODUCTION}

Increased nutrient loads from catchments over the past 200 years are believed to be a driving force behind the spread of Crown of Thorns Starfish, which has been responsible for massive damage to ecosystems in the Great Barrier Reef Lagoon (GBRL) in recent decades (De'ath et al. 2012). Increased nutrient loads also drive increases in pelagic primary production, which can have a complex range of ecological impacts.

A large project (eReefs) is currently underway to implement a system of coupled catchment and marine models for the entire GBRL (Chen et al. 2011). The marine models are implementations of SHOC and EMS (Robson et al. 2008; Margvelashvili et al. 2013; Wild-Allen 2013), which operate on small time-steps and require as input daily or sub-daily estimates of concentrations of sediments, dissolved and particulate nutrients in each of 22 rivers flowing into the system.

Semi-distributed models that predict average annual loads of sediments, nitrogen and phosphorus in GBR catchments have been implemented in Source Catchments and its predecessors (McCloskey et al. 2011), but have so far not demonstrated the ability to accurately simulate day-to-day variations in the concentrations of nutrients.

Recently, Generalised Additive Models (GAMs) such as LRE (“Load Regression Estimator”) have been developed and implemented for several GBRL rivers (Wang et al. 2011; Kuhnert et al. 2012). GAMs are generalised linear models which incorporate smooth functions, $s()$ of covariates. These functions do not have a pre-defined form, but attempt to capture the main features of the data. GAMs have been demonstrated as a powerful tool for prediction of sediment loads, requiring much less input data and lower computational costs than process-based models. LRE has been extended to estimate total nutrient as well as sediment loads from Great Barrier Reef catchments (Kroon et al. 2012) to provide a firm comparative basis for management.

The LRE model is simply defined as:

$$
\log \left(c_{i}\right)=\beta_{0}+\sum_{k=1}^{p} \alpha_{k} x_{k i}+\sum_{k=1}^{m} s_{k}\left(z_{k i}\right)+\varepsilon_{i}
$$

where $c_{i}$ is the concentration of a constituent at time $i, x_{k i}$ and $z_{k i}$ are covariates measured at that time, $s_{k}()$ represents a spline that fits a flexible smoothing function to the data and $\varepsilon_{i}$ is a normally distributed error term. $x_{1 i}$ to $x_{3 i}$ represent linear and quadratic flow terms and a categorical term to indicate whether flow is rising or falling. Smoothed terms $\left(z_{k i}\right)$ include discounted flow terms (discussed below). Additional covariates, which may or may not be log terms, are included on a case-by-case basis (Kuhnert et al. 2012).

Although LRE achieves good agreement with observed sediment, total nitrogen and total phosphorus concentrations in both the Burdekin River (Kuhnert et al. 2012) and Fitzroy River (our analysis), when the method is applied to prediction of dissolved organic or inorganic nutrients on daily time-steps, LRE does not achieve satisfactory results if driven by flow and discounted flow alone.

In this paper, we apply LRE using a range of additional covariates to the Fitzroy River, one of the largest rivers flowing into the GBRL and demonstrate models that provides good agreement with observational measurements of dissolved inorganic and organic nitrogen and phosphorus as well as sediment and particulate nutrient concentrations in the Fitzroy River.

\section{METHODS}

The dataset used to develop our model includes 102 Total Suspended Solids (TSS) records and 67 nutrient records, from samples taken in the lower Fitzroy River under varying flow conditions between 2003 and 2008. An event sampling strategy was followed, so most measurements relate to flow events. Nutrients measured include total phosphorus (TP), total dissolved phosphorus (TDP), particulate phosphorus (PP, measured as the difference between TP and TDP), dissolved inorganic phosphorus (DIP), dissolved organic phosphorus (DOP), total organic phosphorus (TOP), total nitrogen (TN), particulate nitrogen (PN), dissolved organic nitrogen (DON), total organic nitrogen (TON), and dissolved inorganic nitrogen (DIN), comprising ammonia (NH3) and nitrogen oxides (NOx). In order to arrive at a first-order estimate of particulate organic nutrient concentrations, we assumed that inorganic components of particulate nitrogen and phosphorus were negligible in comparison with Particulate Organic Phosphorus (POP) and Particulate Organic Nitrogen (PON). Data from a further 127 samples taken between November 1993 and July 2012 were held back for validation.

Meteorological data were obtained from a Bureau of Meteorology monitoring site at Yeppoon. 
Robson and Dourdet, Incorporating a generalised additive model of river nutrient concentrations into a mechanistic receiving water model

In developing our models, we considered a range of potential hydrological and meteorological control variables that are routinely monitored, available through web services in near real-time, and were considered to have a potential influence on sediment or nutrient concentrations. Each is discussed in turn below.

- Flow: Stage height is monitored continuously and converted to an estimate of flow. Flow is the driving force in the transport of sediments and nutrients from catchments. The flow record was almost complete, but has been interpolated across short intervals where necessary to match times of water quality samples.

- Flow in tributaries: As each tributary is slightly different in character, size and land use, it is to be expected that each will contribute a different proportion of sediments and nutrients for a rainfall event of similar size. Tributaries include the Nogoa, Comet and Isaac Rivers, among others.

- Date (days since the beginning of the flow record) was included as a proxy for seasonal and temporal effects not otherwise captured. This term has been found useful by previous authors (Kuhnert, 2012).

- Wind speed was included for its potential role in sediment resuspension in low flow conditions.

- Barometric pressure: Changes in air pressure may act as a proxy for localised storm events.

- Water temperature influences rates of biological and chemical processes acting on nutrients.

- Air temperature could be an effective proxy for water temperature, with a more reliable record.

- Electrical conductivity (EC) is affected by soil type, land use and interactions between surface and groundwater. It was hypothesised that EC may therefore act as a convenient proxy for the influence of these variables on dissolved nutrient concentrations in particular.

- The rising-falling limb term (RF) indicates whether flow is increasing or decreasing in time. When flow is increasing, concentrations of suspended and dissolved materials are likely to be higher than at the same flow on the falling limb (later in a flow event). We follow Kuhnert et al. (2012) in (2), where $Q_{i}$ is flow at time $i$ and $\mathrm{Q}^{[90]}$ is the 90th percentile flow:

$$
R F=\left\{\begin{array}{cr}
1 & \text { if } Q_{i}>Q_{i-1} \text { and } Q_{i}>Q^{[90]} \\
-1 & \text { if } Q_{i}<Q_{i-1} \text { and } Q_{i}>Q^{[90]} \\
0 & \text { otherwise }
\end{array}\right.
$$

- Discounted flow (DF) represents a weighting of flow history designed to allow the recent prior flow volume to influence concentration predictions (Wang et al. 2011). For example, events that occur after a lengthy dry period tend to result in higher transport of sediment and nutrients due to a higher amount of readily transported material having accumulated in the catchment. Here, we specify:

$$
D F(d)=\sum_{i=1}^{j} d^{j+1-i} Q_{i} / \sum_{i=1}^{j} d^{j+1-i}
$$

where $\mathrm{d}$ is the discount factor $(0<\mathrm{d}<1)$. As $d$ approaches $1, D F$ approaches the mean flow. As $d$ approaches $0, D F$ approaches the instantaneous flow. We considered values of $d$ between 0.2 (close to the long-term mean) and 0.99 (close to the instantaneous flow). Of the four discount factors tested, a discount factor (d) of 0.9 was found to yield the best prediction of sediments, so results are not shown for alternative values of DF.

GAMs were developed and tested in $\mathrm{R}$ ( $\mathrm{R}$ Core Team 2012) using the mgcv package (Wood 2011). Assumptions underlying the application of GAMs were validated following the advice of Zuur et al. (2009). This included checking for auto-correlation in the residuals, normality, heteroscedascity, etc. Spline terms were constrained to a maximum of 4 to 5 knots, to limit the amount of "wriggle" in the fits. In many cases, $\log$ transforms were required to stabilise variance.

\section{RESULTS}

All models reported in Table 1 to Table 3 showed acceptable agreement with the assumptions of normality, homoscedacity and linearity, and include only significant covariate terms. Neither meteorological variables, electrical conductivity nor the rising-falling limb term were found to be useful predictive terms, so candidate models including these terms are excluded from further consideration. Other input variables are included in tables only when they are found to be significant. Cross-validation to assess goodness of fit was achieved for all candidate models by comparing observations with in-sample predictions in the first instance (e.g. Figure 2).

\subsection{Sediments}

Table 1 shows model statistics for three candidate models. Water temperature does not significantly improve the GAM at $\mathrm{p}<0.05$. While including date in the GAM did improve the match between observational data and model predictions, it did not reveal clear seasonal patterns and was excluded from the final model because we 
Robson and Dourdet, Incorporating a generalised additive model of river nutrient concentrations into a mechanistic receiving water model

were not confident that the unexplained temporal patterns in our limited dataset could be expected to persist in future.

Including flow in Comet and Isaac Rivers (TSS3) slightly improved the percent of deviance explained, but the improvement came at a cost reduced significance of the fit (Table 1 ), so this model was not chosen as the preferred TSS model. Flows in other tributaries were not significant terms; nor was the rising-falling limb term.

\subsection{Particulate nutrients}

Particulate nutrients (PN and PP) in a river are a component of total suspended solids, are subject to the same physical transport processes and can be predicted either directly from TSS (PN1 in Table 2) or using the same model terms used to predict TSS (PN2). Using smoothing rather than a log transform for flow (PN3) provides a better fit again, with similar validation results. Including the date term (PN4) improves the fit slightly, but this term should be used with caution as the causal factor behind this relationship is unknown and may not continue in the future. All other terms included have wellknown or hypothesised relationships with sediment and nutrient concentrations (Dourdet and Robson, submitted). Due to space restrictions, nitrogen but not phosphorus results are shown here.

In the case of PP but not PN, including flow in Connors River and Isaac River substantially improves the results without reducing the significance of the fit. Meteorological observations and electrical conductivity were not significant predictive variables. Water temperature was significant, but improved results by such a small margin that it was not considered worth including in the final models.

\subsection{Dissolved nutrients}

Summary statistics for several candidate models for dissolved inorganic nitrogen are presented in Table 3. Similar results were found for dissolved organic nitrogen and dissolved phosphorus constituents. In general, the predictive power of models for dissolved nutrients was weaker than those for particulate materials unless a date smoother term was included (models DIN2), but despite achieving high $\mathrm{r}^{2}$ and

Table 1. Fit statistics for models to predict TSS. GAM terms are given in the format of the R statistical programming language.

\begin{tabular}{|c|c|c|c|c|c|}
\hline $\begin{array}{l}\text { Model } \\
\text { Name }\end{array}$ & GAM terms & Term & $\mathbf{p}$ & $\begin{array}{l}\text { Deviance } \\
\text { explained } \\
\text { (DE) }\end{array}$ & adjusted $r^{2}$ \\
\hline TSS1 & $\log (\mathrm{TSS}) \sim \log$ (flow) & $\log$ (flow) & $<0.001$ & $13.5 \%$ & 0.13 \\
\hline \multirow[t]{2}{*}{ TSS2 } & \multirow[t]{2}{*}{$\log (\mathrm{TSS}) \sim \log ($ flow $)+\mathrm{s}(\mathrm{DF})$} & $\log$ (flow) & $<0.001$ & \multirow[t]{2}{*}{$53.4 \%$} & \multirow[t]{2}{*}{0.52} \\
\hline & & $\begin{array}{l}\mathrm{s}(\mathrm{DF} \\
\mathrm{d}=0.9)\end{array}$ & $<0.001$ & & \\
\hline \multirow[t]{4}{*}{ TSS3 } & \multirow{4}{*}{$\begin{array}{l}\log (\text { TSS }) \sim \log (\text { flow })+s(\text { DF }) \\
+\log (\text { Comet })+\log (\text { Isaac })\end{array}$} & $\log$ (flow) & $<0.01$ & \multirow[t]{4}{*}{$70.5 \%$} & \multirow[t]{4}{*}{0.64} \\
\hline & & $\begin{array}{l}\mathrm{s}(\mathrm{DF}, \\
\mathrm{d}=0.9)\end{array}$ & $<0.01$ & & \\
\hline & & $\log ($ Comet $)$ & $<0.05$ & & \\
\hline & & $\log$ (Isaac) & $<0.05$ & & \\
\hline
\end{tabular}

Table 2. Statistical summary of several candidate models for particulate nitrogen. GAM terms are given in the format of the $\mathrm{R}$ statistical programming language. The final preferred model is highlighted. Only models with $\mathrm{p}<0.05$ are shown. $\mathrm{p}$ values correspond to $t$ statistics for parametric terms, F statistics for smoothed terms.

\begin{tabular}{|c|c|c|c|c|c|}
\hline $\begin{array}{l}\text { Model } \\
\text { Name }\end{array}$ & GAM terms & Term & $\mathbf{p}$ & $\begin{array}{c}\text { Deviance } \\
\text { explained } \\
\text { (DE) }\end{array}$ & adjusted $r^{2}$ \\
\hline PN1 & $\mathrm{PN} \sim \mathrm{TSS}$ & TSS & $<0.001$ & $61.0 \%$ & 0.60 \\
\hline \multirow[b]{2}{*}{ PN2 } & \multirow{2}{*}{$\begin{array}{l}\log (\mathrm{PN}) \sim \log (\text { flow }) \\
+\mathrm{s}(\mathrm{DF})\end{array}$} & $\log$ (flow) & $<0.05$ & \multirow[b]{2}{*}{$48.4 \%$} & \multirow[b]{2}{*}{0.45} \\
\hline & & $\begin{array}{l}\mathrm{s}(\mathrm{DF} \\
\mathrm{d}=0.9)\end{array}$ & $<0.001$ & & \\
\hline \multirow[b]{2}{*}{ PN3 } & \multirow{2}{*}{$\begin{array}{l}\log (\mathrm{PN}) \sim \mathrm{s}(\text { flow }) \\
+\mathrm{s}(\mathrm{DF})\end{array}$} & $\mathrm{s}$ (flow) & $<0.001$ & \multirow[b]{2}{*}{$59.5 \%$} & \multirow[b]{2}{*}{0.56} \\
\hline & & $\begin{array}{l}\mathrm{s}(\mathrm{DF}, \\
\mathrm{d}=0.9)\end{array}$ & $<0.001$ & & \\
\hline \multirow{3}{*}{ PN4 } & \multirow{3}{*}{$\begin{array}{l}\log (\mathrm{PN}) \sim \mathrm{s}(\text { flow }) \\
+\mathrm{s}(\mathrm{DF})+\mathrm{s}(\text { date })\end{array}$} & $\log$ (flow) & $<0.001$ & \multirow{3}{*}{$64.0 \%$} & \multirow{3}{*}{0.60} \\
\hline & & $\begin{array}{l}\mathrm{s}(\mathrm{DF}, \\
\mathrm{d}=0.9)\end{array}$ & $<0.001$ & & \\
\hline & & s(date) & $<0.05$ & & \\
\hline
\end{tabular}


Robson and Dourdet, Incorporating a generalised additive model of river nutrient concentrations into a mechanistic receiving water model

$\mathrm{p}<0.05$ for the in-sample training data, models that included date terms performed poorly when evaluated against an independent data set.

While a discount factor of 0.9 provided the best results for TSS, PN and PP, for dissolved nutrients, a discount factor of 0.95 (weighting towards more recent flows) yielded better results. For dissolved nutrients, the $\log$ (flow) or s(flow) term was not significant when included in addition to discounted flow, and yielded a poorer fit to observations when included instead of discounted flow.

Inclusion of flow in tributaries dramatically improved the results for dissolved inorganic and organic nutrients, with flow in the Nogoa River found to be the strongest predictor for most dissolved nutrient species. In the case of DIN, flow in Isaac River proved a slightly stronger predictor than flow in Nogoa River (model DIN4 vs. DIN3). Nogoa River is not a significant term when Isaac River flow is also included: the two are highly correlated.

For DOP, satisfactory results could not be achieved without inclusion of tributary flow, and date was not a significant term if included in the GAM. Including both Nogoa and Comet Rivers along with discounted flow in the Fitzroy River yielded the best results.

The predictive power of all final, preferred models was cross-validated by application to a period outside that used to develop the models, using as input all flows between November 1993 and July 2012 that were not included in the original 2003-2008 dataset. Results for sediments and TN are illustrated in Figure 3. While results for the validation dataset are not as accurate as those for the in-sample fitting dataset, suggesting either some over-fitting or a shift in catchment conditions, the models nonetheless provided a useful degree of predictive power, performing better than the available alternative methods of specifying inflow concentrations for marine models.

\section{DISCUSSION}

This study provides further evidence that GAMs can provide greatly improved predictions of concentrations of particulate materials (total suspended solids, particulate nitrogen and particulate phosphorus) at a daily time-step in comparison with simple linear regressions that rely only on flow as a predictive variable. These approaches have been previously demonstrated for Great Barrier Reef

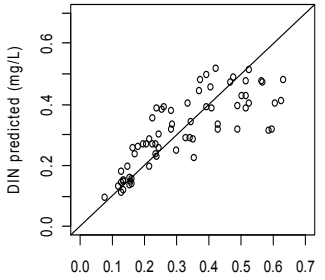

DIN obsened (mg/L)

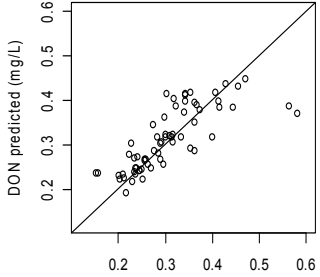

DON obsened (mg/L)

Figure 2. In-sample prediction results for dissolved inorganic nitrogen (DIN, model DIN4) and dissolved organic nitrogen.
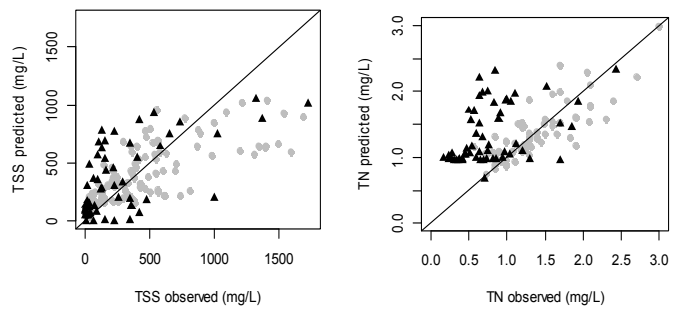

Figure 3. Comparison of GAM results using data held back from the original model fitting procedure (black triangles, out-of sample prediction) and data included in the fitting procedure (in-sample, grev). rivers, including the Burdekin River, by Wang et al. (2010), Kuhnert et al. (2012) and Kroon et al. (2012). The present study shows that similar models apply to materials in the Fitzroy River.

The enhanced models demonstrated here can be used to predict concentrations of dissolved nutrients, which are not amenable to prediction from flow in the main river channel alone. Unlike particulate nutrient concentrations, dissolved nutrient concentrations are not strongly related to sediment concentrations, and do not exhibit an obvious relationship to flow.

In the course of developing these models, we explored the potential impacts of several additional input variables which have not been considered by previous authors: wind velocity, air temperature and barometric pressure, water temperature, electric conductivity, and flow in tributaries. These variables were selected for consideration in part because of plausible causative relationships with variations in the target variables, and in part because they are easily and routinely measured at high frequency, and the results are available online in near-real time. This makes them suitable for inclusion in models that will be required to run routinely in near-real time.

Flow in tributaries and water temperature were both to improve the predictive power of the models. While tributary flow was found to be important, the significance of water temperature was low in comparison with that of the other variables included in the models and its inclusion added only marginally to their predictive power. The other new variables considered (meteorological measurements and electrical conductivity) were 
Robson and Dourdet, Incorporating a generalised additive model of river nutrient concentrations into a mechanistic receiving water model

not found to be significant. This may in part be due to the use of instantaneous values: some covariates, such as air pressure, may cause a response on a time-scale of a few days. Further exploration considering various response time-scales may reveal additional predictive relationships.

Kunhert et al. (2012), in their study of the Burdekin River, suggested that the rising-falling limb term is not significant in case of large rivers. Our study confirmed this in the case of the Fitzroy River. Though Kunhert et al. (2012) shows that inclusion of a seasonal term improves prediction of sediment and nutrients loads, we were unable to include this variable due to insufficient dry-season observational data for the Fitzroy River.

Although including the date as a predictive variable increased the percent deviance explained by most of the models, where possible, we have preferred models that do not include time as a determinant. Our dataset is relatively small and apparent relationships involving time over the period of our data may not hold true when the model is applied over longer periods. When date is included, it is found to be insignificant for TSS, significant for particulate nutrients and very significant for dissolved nutrients.

Using a discounted flow term as well as a flow term considerably improves all the models. However, contrary to the findings of Kunhert et al. (2012) for the Burdekin River, combining several discounted flow terms did not improve our models as only one discounted flow term proved to be significant for any given model: there was significant co-linearity amongst different DF terms.

The optimal discount factor was found to depend on which category of variable we are interested in. A discount factor of 0.9 gave the best results in the case of sediments and particulate nutrients, while dissolved nutrients are better predicted by a discount factor of 0.95 . This difference suggests that dissolved nutrients are affected by flow over longer time-scales than are particulate materials. Particulate materials eroded from catchments and river banks or suspended from the river bed during high flows may settle to the bottom as flow recedes, while dissolved materials remain in the water column.

There is strong correlation between TSS and particulate nutrients (because particulate nutrients are a component of TSS), such that a simple log-linear regression between TSS and PP provides a deviance explained of $77 \%$, and between TSS and PN, a deviance explained of $60 \%$. GBR catchment soils naturally contain substantial stocks of nitrogen and phosphorus (Furnas, 2003). To provide models useful for predictive purposes, however, it was desirable to build models for nutrient concentrations that did not depend on TSS, which is not monitored in real-time.

Flow in tributaries (specifically, the Nogoa River, Comet, Isaac and Connors Creek) was found to substantially improve predictive capacity, particularly in the case of dissolved nutrients. This reflects the different land-uses and geological characteristics of the different sub-catchments and highlights the key role of Nogoa River in determining dissolved nutrient concentrations in the Fitzroy River. The catchment of Nogoa River is dominated by cattle pasture, so one might speculate that animal manure contributes to the dissolved nutrient load in this system. Tributary flow is a component of overall flow terms, and inclusion of both may adversely affect the error structure of the model. Multi-variate time-series analysis may help to disentangle relationships between the various covariate and predictor variables.

\section{CONCLUSIONS}

We have extended the approach of previous authors to consider several additional potential forcing terms using data available from routine monitoring in the Fitzroy River and a nearby meteorological station. Of the additional terms considered, only tributary flow from the Nogoa River was found to significantly improve the model. This additional term, however, greatly improved results for dissolved nitrogen and phosphorus concentrations, allowing these to be predicted on a daily time-step for the first time.

The results highlight the importance of a particular sub-catchment (the Nogoa) in determining dissolved inorganic nitrogen and phosphorus concentrations reaching the river mouth. This insight may direct attention in further development of process-based models of the catchment to facilitate long-term scenario evaluation. Meanwhile, combining GAM with a deterministic catchment model (Source Catchments) to predict changes in sediment and nutrient loads and a detailed mechanistic receiving water model (EMS) provides a way forward for scenario modelling to support management decisions.

The time series produced from GAM outputs do not capture the error term and thus tend to underestimate the variability of sediment and nutrient concentrations. Future work may include incorporating both the GAM results and the mechanistic marine model in a Bayesian hierachical framework to better understand the effects of errors in both. 
Robson and Dourdet, Incorporating a generalised additive model of river nutrient concentrations into a mechanistic receiving water model

\section{ACKNOWLEDGMENTS}

This work was supported by the CSIRO Wealth from Oceans National Research Flagship and the eReefs collaboration, which receives funding from the Great Barrier Reef Foundation and the Science Innovation Endowment Fund (SIEF). One of the authors (Mr Vincent Dourdet) was an unpaid industrial trainee at CSIRO Land and Water: this work was conducted as part of the requirements for completion of a Bachelor of Engineering degree at the Ecole des Mines, d'Ales, France. Thanks to Ms Aurelie Mousques for assistance in formatting this manuscript. An extended version of this paper has been submitted for consideration by Environmental Modelling \& Software (Dourdet and Robson, submitted).

\section{REFERENCES}

Chen, Y., S. Seaton, S. A. Minchin, K. D. Joehnk, B. J. Robson and Q. B. (2011). eReefs Pilot Report. Canberra: 72.

De'ath, G., K. A. Fabricius, H. Sweatman and M. Puotinen (2012). "The 27-year decline of coral cover on the Great Barrier Reef and its causes." Proceedings of the National Academy of Sciences of the United States of America 109(44): 17995-17999. 10.1073/pnas.1208909109.

Kroon, F. J., P. M. Kuhnert, B. L. Henderson, S. N. Wilkinson, A. Kinsey-Henderson, B. Abbott, J. E. Brodie and R. D. R. Turner (2012). "River loads of suspended solids, nitrogen, phosphorus and herbicides delivered to the Great Barrier Reef lagoon." Marine Pollution Bulletin 65(4-9): 167-181. DOI 10.1016/j.marpolbul.2011.10.018.

Kuhnert, P. M., B. L. Henderson, S. E. Lewis, Z. T. Bainbridge, S. N. Wilkinson and J. E. Brodie (2012). "Quantifying total suspended sediment export from the Burdekin River catchment using the loads regression estimator tool." Water Resources Research 48Doi 10.1029/2011wr011080.

Margvelashvili, N., J. Andrewartha, M. Herzfeld, B. J. Robson and V. E. Brando (2013). "Satellite data assimilation and estimation of a 3D coastal sediment transport model using error-subspace emulators." Environmental Modelling \& Software 40(0): 191-201. DOI 10.1016/j.envsoft.2012.09.009.

McCloskey, G., R. Ellis, D. Waters and J. Stewart (2011). PEST hydrology calibration process for source catchments-applied to the Great Barrier Reef, Queensland. 19th International Congress on Modeling and Simulation, Perth, Australia

R Core Team (2012). R: A Language and Environment for Statistical Computing. Vienna, Australia, R Foundation for Statistical Computing

Robson, B. J., D. P. Hamilton, I. T. Webster and T. Chan (2008). "Ten steps applied to development and evaluation of process-based biogeochemical models of estuaries." Environmental Modelling \& Software 23(4): 369-384. DOI 10.1016/j.envsoft.2007.05.019.

Wang, Y. G., P. Kuhnert and B. Henderson (2011). "Load estimation with uncertainties from opportunistic sampling data - A semiparametric approach." Journal of Hydrology 396(1-2): 148-157. DOI 10.1016/j.jhydrol.2010.11.003.

Wild-Allen, K. (2013). "Mechanisms driving estuarine water quality: A 3D biogeochemical model for informed management." Estuarine, coastal and shelf science10.1016/j.ecss.2013.04.009.

Wood, S. N. (2011). "Fast stable restricted maximum likelihood and marginal likelihood estimation of semiparametric generalized linear models." Journal of the Royal Statistical Society Series BStatistical Methodology 73: 3-36. DOI 10.1111/j.1467-9868.2010.00749.x.

Zuur, A. F. (2009). Mixed effects models and extensions in ecology with R. Statistics for biology and health. New York, NY, Springer 